
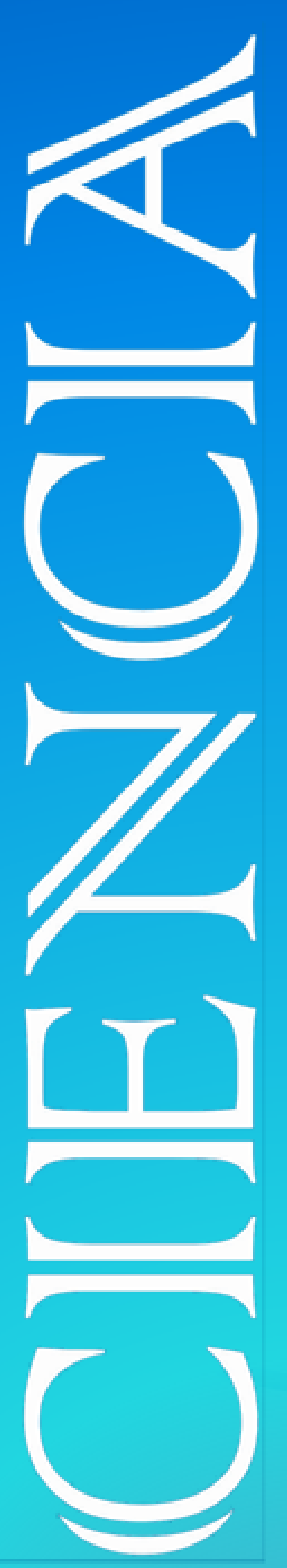

Esta publicación cientfica en formato digital es continuidad de la revistaimpresa

Depósito Legal: pp 199302Z2U47 ISSN:1315-2076
Depósito Legal ppi $201502 Z 44668$

\section{Vol. 24, $\mathbf{N}^{\circ} 2$ Abril - Junio 2016}

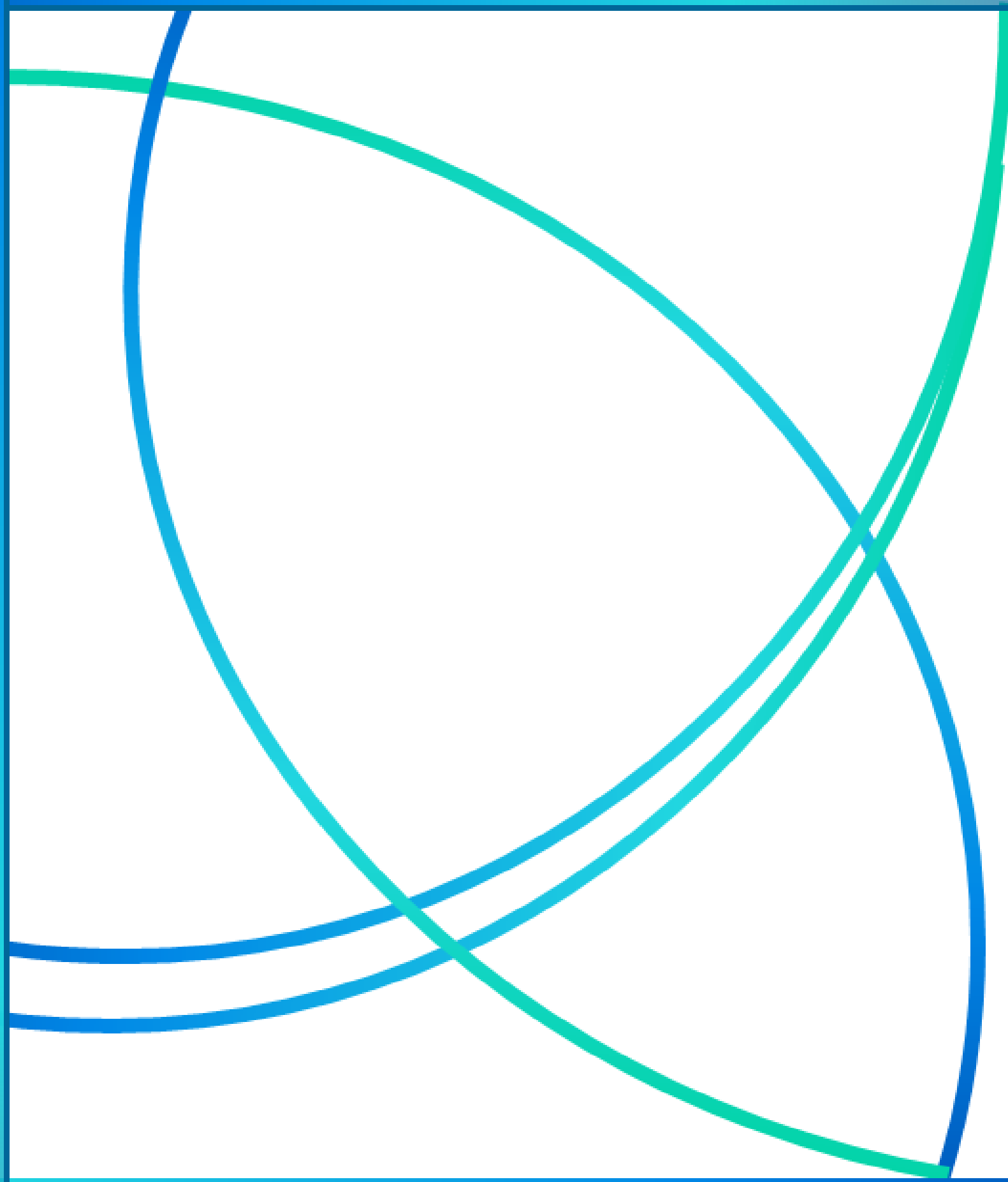

An International Refereed Scientific Journal of the Facultad Experimental de Ciencias at the Universidad del Zulia 


\title{
Especiación del fósforo en sedimentos de un río tropical venezolano y su posible impacto sobre el ecosistema
}

\author{
Aristide Márquez ${ }^{1, *}$, Ángel González², William Senior' ${ }^{1}$ y Antonio \\ Benítez ${ }^{1}$ \\ ${ }^{1}$ Departamento de Oceanografía, Instituto Oceanográfico de Venezuela, \\ Universidad de Oriente. \\ ${ }^{2}$ Departamento de Recursos Acuáticos, Instituto Limnológico, Universidad \\ de Oriente, Núcleo Bolívar, Venezuela
}

Recibido: 15-12-15 Aceptado: 28-04-16

\section{Resumen}

En este estudio, se empleó una modificación del método de extracción sedimentaria (SEDEX) para cuantificar las diferentes formas de $\mathrm{P}$ en los sedimentos recolectados en río Cuchivero durante marzo del año 2014. El rango de variación de las concentraciones de las diferentes formas de $\mathrm{P}$ respecto al $\mathrm{P}$ total $\left(\mathrm{PT}_{\mathrm{f}}\right)$ fueron 4,84-7,49 $\mu \mathrm{gP} / \mathrm{g}$ para la fracción $\mathrm{F} 1$ constituida por el $\mathrm{P}$ intercambiable ( $\mathrm{P}-\mathrm{ad})$ más el asociado a óxidos de hierro $(\mathrm{P}-\mathrm{Fe})$ con mayor preponderancia del P-Fe; 0,58-1,61 $\mu \mathrm{gP}$-autigénico/g (F2); 2,03-6,38 $\mu \mathrm{g} \mathrm{P}$ detrítico/g (F3), y 28,59-68,99 $\mu \mathrm{g}$ P-orgánico/g (F4), respectivamente. Estos valores representan en promedio 11, 54\%; 1,62\%; 7,67\% y 79,08\% para las cuatro fracciones respectivamente. El fósforo orgánico fue la forma más representativa de la especiación de $\mathrm{PT}_{\mathrm{f}}$. El porcentaje promedio de $\mathrm{P}$ biodisponible representado por $\mathrm{F} 1+\mathrm{F} 4$ fue 52,72 $\mu \mathrm{g} \mathrm{P} / \mathrm{g}$, lo cual representa el 90,63\% del $\mathrm{PT}_{\mathrm{f}}$. La distribución de las diferentes formas de $\mathrm{P}$ en los sedimentos mostró asociación con el menor tamaño de la partícula y diferentes mecanismos posdeposicionales que pueden ser afectados por las fuentes de materiales de entrada, tamaño de grano, ambientes de depósito, condiciones hidrológicas y las actividades antropogénicas.

Palabras clave: especiación, fósforo, sedimento, río tropical, Venezuela.

Phosphurus speciation in sediments of a Venezuelan tropical river and its posible impacto $\mathrm{n}$ the ecoystem

\begin{abstract}
In this study, a modification of the method of extraction sedimentary (SEDEX) was used to quantify the different forms of $\mathrm{P}$ in sediments collected in Cuchivero river during March 2014. The range of variation of the concentrations of the different forms of $\mathrm{P}$ respect the total $\mathrm{P}\left(\mathrm{TP}_{\mathrm{f}}\right)$ were $4.84-7.49 \mu \mathrm{gP} / \mathrm{g}$ to the $\mathrm{F} 1$

\footnotetext{
*Autor para la correspondencia: aristd@gmail.com
} 
fraction consisting of exchangeable $\mathrm{P}$ (ad-P) plus associated with iron oxides (Fe-P) with greater preponderance of $\mathrm{P}-\mathrm{Fe} ; 0,58-1,61 \mu \mathrm{gP} / \mathrm{g}$ - authigenic (F2); 2.03 - 6.38 $\mu \mathrm{gP} / \mathrm{g}$ - detrital (F3), and 28.59 - $68.99 \mu \mathrm{gP} / \mathrm{g}$-organic (F4), respectively. These values represent promedio11, 54\%; $1.62 \% ; 7.67 \%$ and $79.08 \%$ respectively for the four fractions. The organic phosphorus was the way most representative fraction speciation of $\mathrm{TP}_{\mathrm{f}}$. The average percentage of bioavailable $\mathrm{P}$ represented by $\mathrm{F} 1+\mathrm{F} 4$ fraction of $\mathrm{P}$ was $52.72 \mu \mathrm{g}-\mathrm{P} / \mathrm{g}$, which represents $90.63 \%$ of the $\mathrm{TP}_{\mathrm{f}}$. The distribution of the different forms of $\mathrm{P}$ in sediments showed association with smaller particle size and postdepositional different mechanisms may be affected by sources of input materials, grain size, depositional environments, hydrological conditions and anthropogenic activities.

Keywords: speciation, phosphorus, sediment, tropical river.

\section{Introducción}

El fósforo (P) es un macronutriente que existe en muchas formas en ambientes acuáticos y que limita la productividad en la mayoría de las zonas fluviales, estuarinas, y costeras (1). El principal mecanismo por el cual el fósforo es introducido a los ríos es debido a la meteorización de apatita y fosforitas, así como de actividades antropogénicas como la agricultura y en los océanos se incorpora a través del $\mathrm{P}$ disuelto proveniente de las fuentes terrígenas como apatita y fosforitas $(2,1,3)$. Sin embargo, las concentraciones han aumentado desde el siglo pasado debido a la erosión y a los aumentos acelerados de las fuentes antropogénicas en los suelos de los continentes, causando un desequilibrio y generando problemas de eutrofización en las zonas fluviales y marino-costeras $(4,5)$.

En sedimentos de ríos, lagos, estuarios y océanos, el $\mathrm{P}$ existe en forma orgánica e inorgánica, siendo controlada su movilidad y biodisponibilidad por la asociación con minerales, especialmente por adsorción con hierro, calcio, aluminio, manganeso, y por la de ciertas fracciones orgánicas recalcitrantes (6, 7).

Parte del fósforo que interviene en el ciclo biogeoquímico, queda inmovilizado en los sedimentos en parte como fosfato de calcio o como fosfato asociado a hierro, también removido por procesos inorgánicos como adsorción sobre superficies reactivas como arcillas y oxihidróxidos de hierro, carbonatos y por la formación de fases minerales autigénicas como apatita (8). Debido a que en los ríos Cuchivero y Guaniamo, Municipio, Cedeño, estado Bolívar, no existen investigaciones relacionadas con fraccionamiento de fósforo en los sedimentos, y que este elemento juega un papel importante es la productividad de los ecosistemas acuáticos, se planteó como objetivo, cuantificar y estudiar la distribución espacial de las fracciones, para aportar la primera información sobre el aporte al fósforo total, de las fracciones intercambiable o lábil ( $\mathrm{P}$ ad) más fósforo asociado a óxidos de 
hierro (P-Fe), representado por la fracción $\mathrm{F}$ 1, el fósforo autigénico representado por la fracción F2, y el fósforo detrítico y orgánico simbolizados por las fracciones F3 y F4 respectivamente.

Por otra parte, este estudio permitirá discernir sobre el posible reacomodo deposicional del $\mathrm{P}$ en los sedimentos de estos ecosistemas. En tal sentido se utilizó el método de extracción secuencial sedimentaria (SEDEX) modificado por Anderson y Delaney (9) para cuantificar los cuatro reservorios antes descritos.

\section{Materiales y métodos}

El área de estudio se ubicó en un transepto aproximado de $55 \mathrm{~km}$ en el río Cuchivero, el cual está localizado hacia el sur de Venezuela, específicamente en el estado Bolívar. El Cuchivero nace en la vertiente noroeste de la altiplanicie de Icutú, en la serranía de Guamapí de la sierra de Maigualida, tiene una longitud de 305 kilómetros, y drena una cuenca de aproximadamente $16.000 \mathrm{~km}^{2}$ de extensión y desemboca en el Orinoco, aguas abajo de la ciudad de Caicara del Orinoco. En su recorrido recibe,

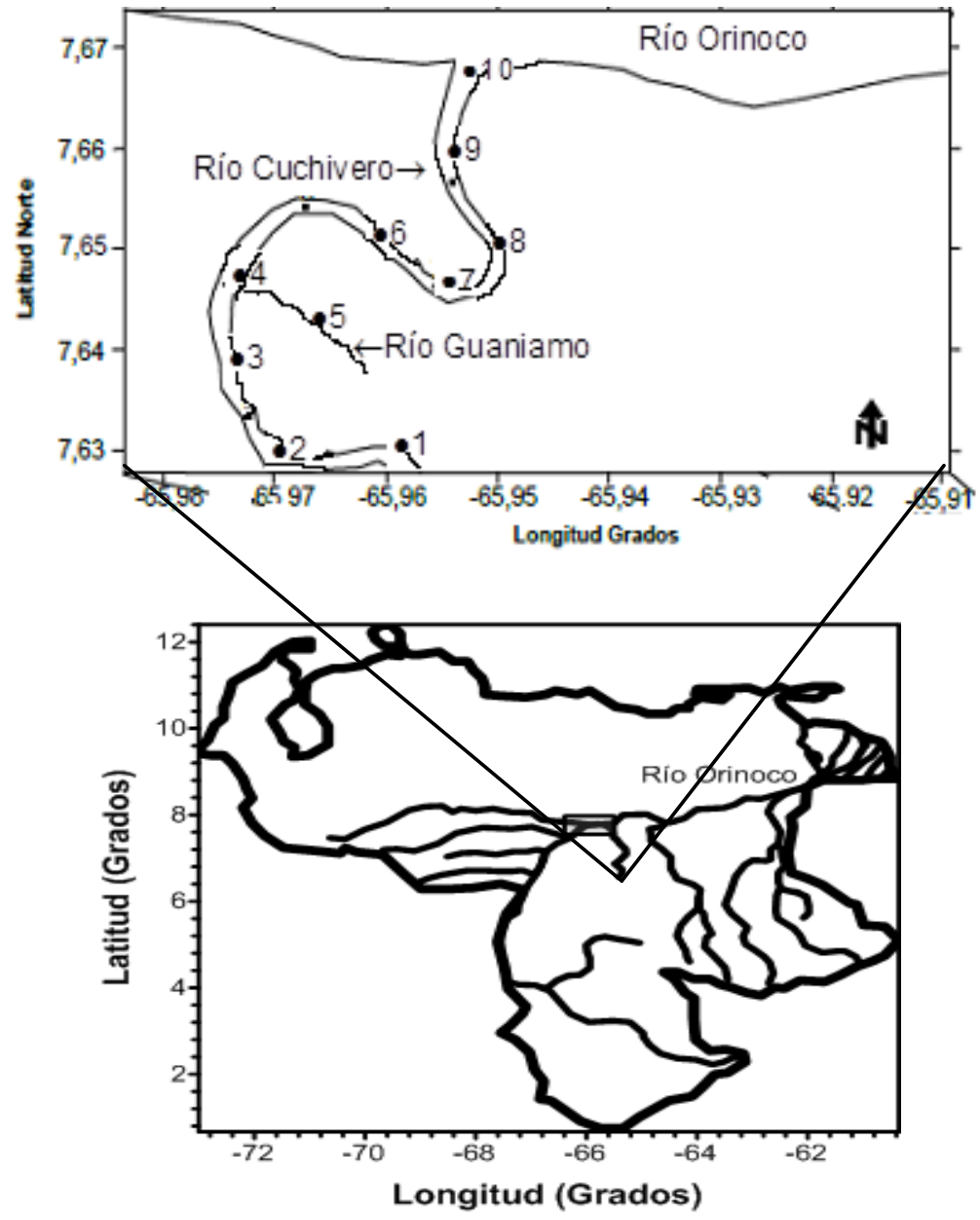

Figura 1. Zona de estudio mostrando las díez estaciones de recolección de las muestras en el río Cuchivero y Guaniamo, Venezuela 
por el oeste o margen izquierda, al río Guaniamo, su principal afluente (10). En marzo del año 2014, se recolectaron sedimentos superficiales en diez estaciones de muestreo aguas abajo a lo largo del río Cuchivero, desde la localidad de Puerto Matú en la cual se encuentran las minas de diamantes (estación 1), hasta su desembocadura en el río Orinoco (estación 10). Del grupo de estaciones muestreadas, se recolectó una muestra dentro del río Guaniamo (estación 5). Los sedimentos se recolectaron utilizando una draga tipo Eckman de 0,02 $\mathrm{m}^{2}$ de área. Con el apoyo de una paleta plástica las muestras se colocaron en frascos de polipropileno de $250 \mathrm{ml}$ de capacidad previamente rotulados y lavados con una solución ácida ( $\mathrm{HCl} 1 \mathrm{~mol} / \mathrm{l}) \mathrm{y}$ agua desionizada, calidad Nanopure de conductividad de $18 \mathrm{M} \Omega / \mathrm{cm}$. Las muestras luego se preservaron bajo congelación a $-20^{\circ} \mathrm{C}$.

Los sedimentos preservados fueron descongelados y secados a $60{ }^{\circ} \mathrm{C}$ en una estufa hasta obtener un peso constante, luego se tamizaron para obtener la fracción menor a $2 \mathrm{~mm}$ y se pulverizaron en un mortero de porcelana, para luego ser almacenados en envases de polietileno herméticamente cerrados hasta los análisis.

Se cuantificaron 4 fracciones presentes en el fósforo total. La fracción $\mathrm{F} 1$ (fósforo adsorbido + asociado a óxidos de hierro), autigénico (F2), detrítico (F3) y fósforo orgánico (F4), utilizando el método de Anderson \& Delaney (9). La fracción $\mathrm{F} 1$ fue extraída adicionando a 1 gramo de sedimento,
$10 \mathrm{ml}$ de mezcla citrato de sodio 0,22 $\mathrm{mol} / \mathrm{l}$, ditionito de sodio $0,14 \mathrm{~mol} / \mathrm{l} \mathrm{y}$ bicarbonato de sodio $1 \mathrm{~mol} / \mathrm{l}$ a pH 7,6, luego se agitó por seis horas y se adicionó $10 \mathrm{ml}$ de $\mathrm{MgCl}_{2} 1 \mathrm{~mol} / \mathrm{l} \mathrm{a} \mathrm{pH}$ 8.

Se decantó el líquido sobrenadante para la cuantificación del $\mathrm{P}$ en la fracción $\mathrm{F}$. El residuo se extrajo con $10 \mathrm{ml}$ de acetato de sodio 1 $\mathrm{mol} / \mathrm{l}$ a $\mathrm{pH} 4$ durante dos horas, luego se adicionó $10 \mathrm{ml}$ de $\mathrm{MgCl}_{2} 1 \mathrm{~mol} / \mathrm{l} \mathrm{a}$ pH 8; se agitó durante 2 h, y se agregó $10 \mathrm{ml} \mathrm{de} \mathrm{MgCl}_{2} 1 \mathrm{~mol} / \mathrm{l} \mathrm{a} \mathrm{pH} \mathrm{8,} \mathrm{luego}$ se agitó durante $2 \mathrm{~h}$. El líquido sobrenadante se decantó para la cuantificación de la fracción F2 o antigénica.

El residuo del paso anterior, fue tratado con $13 \mathrm{ml}$ de $\mathrm{HCl} \mathrm{mol} / \mathrm{l} \mathrm{y}$ agitado por $16 \mathrm{~h}$.

El líquido sobrenadante fue decantado y utilizado para la determinación de la fracción $\mathrm{F}_{3}$ o fósforo detrítico. $\mathrm{Al}$ residuo proveniente del paso anterior fue adicionado $1 \mathrm{ml}$ de $\mathrm{MgNO}_{3}$ al $50 \%$ $\mathrm{P} / \mathrm{V}$, luego secado a $80^{\circ} \mathrm{C}$ y sometido a ignición a $550^{\circ} \mathrm{C}$. Las cenizas remanentes se extrajeron posteriormente con $13 \mathrm{ml}$ de $\mathrm{HCl} 1$ mol/l con agitación durante 24 horas para la determinación del fósforo orgánico.

El fósforo total se determinó por la sumatoria de las fracciones extraídas y se confirmó por el método de Valderrama (11) para verificar su exactitud. Para ello, a 0,03 g aproximadamente de sedimento seco contenidos en botellas de vidrio con tapas herméticas, se agregó $50 \mathrm{ml}$ de 
agua desionizada y $3 \mathrm{ml}$ de agente oxidante (persulfato de potasio, ácido bórico e hidróxido de sodio $1 \mathrm{~mol} / \mathrm{l}$ en una proporción 6:4:3,5) y se colocaron en digestión en un autoclave durante media hora a 15 psi de presión y a $270{ }^{\circ} \mathrm{C}$.

La solución del agente sobrenadante se decantó y se determinó el contenido de fósforo. La precisión del método oscila alrededor del $0,20 \%$.

La cuantificación de las diferentes fracciones extraídas, así como el fósforo total, se realizó por colorimetría después del ajuste del $\mathrm{pH}$ a 5,4 usando paranitrofenol como indicador (12).

Todas las mediciones se realizaron de forma automatizada utilizando un auto analizador Technicon II Scientific Instruments AC-100.

Para los análisis del fósforo total se utilizaron patrones certificados de clase analítica ultra pura, Marine Nutrients Standards Kit. La reproducibilidad del método de extracción fue calculada a partir del porcentaje de recuperación (\%), haciendo el cociente entre la sumatoria de todas las fracciones y el PTv.

El método muestra confiabilidad tal como se indica en la tabla 1, en la cual se aprecian porcentajes de recuperación superiores a 98,61\%.

Para el análisis granulométrico, los sedimentos fueron secados a $80^{\circ} \mathrm{C}$ en una estufa P SELECTA y luego pasados través de tamices de 4,00; 2,00; 1,00; o,85; 0,50; 0,25 у 0,063 mm (13).

La materia orgánica total (MOT) fue calculada por diferencia de peso a través del método de calcinación a $540^{\circ} \mathrm{C}$ descrito en (14). Para ello se utilizó una mufla Marca JELRUS y una balanza analítica Marca Denver Instrument M-10 con precisión de $0,0001 \mathrm{~g}$.

Los carbonatos se determinaron por titulación volumétrica con $\mathrm{NaOH}$ 0,50 mol/l (15). Para ello, se pesó $0,50 \mathrm{~g}$ de sedimentos previamente calcinados, se añadió $15 \mathrm{ml}$ de $\mathrm{HCl}$ 0,1 $\mathrm{mol} / \mathrm{l}$ y se calentó hasta ebullición.

Las muestras se filtraron para eliminar la cantidad de sedimento que obstaculizaba el cambio de color del indicador en el punto final de la titulación y se valoró con $\mathrm{NaOH}$ o,29 $\mathrm{mol} / \mathrm{l}$, usando como indicador azul de bromofenol.

La determinación de hierro se realizó por el método descrito en Lewis y Landig (16). $2 \mathrm{~g}$ de sedimento seco, se trató con $10 \mathrm{ml}$ de ácido nítrico, ácido clorhídrico y ácido perclórico concentrado en una relación 3:2:1, utilizando un digestor de Microondas Microdigest 401 de Prolabo a $110^{\circ} \mathrm{C}$, por espacio de 30 minutos y luego se dejó enfriar y se filtró en balones de $25 \mathrm{ml}$.

Los digeridos se cuantificaron utilizando un espectrofotómetro de absorción atómico marca Perkin Elmer 3110 en modo de llama de aire acetileno y corrector de fondo de deuterio. Los análisis de granulometría, materia orgánica y carbonatos se realizaron para determinar las 
asociaciones estadísticas del fósforo con estas variables.

Todos los análisis fueron realizados en el Laboratorio de Química Marina del Departamento de Oceanografía, Instituto Oceanográfico de Venezuela.

Los grados de asociación existentes entre los parámetros se determinaron por análisis estadístico de matriz de correlación de Pearson, utilizando el paquete STATGRAPHICS PLUS 5,1. Los resultados, mínimos, máximos y desviación estándar mostrados en la tabla 1, fueron calculados a partir de los valores de las estaciones del río Cuchivero.

No se incluye el valor de la estación cinco (estación 5), ya que esta corresponde a valores del río Guaniamo.

Tabla 1. Valores de granulometría (AM, AF, Lodos en \%), MOT (\%), $\mathrm{CaCO}_{3}(\%), \mathrm{MOT}(\%)$, hierro $(\mu \mathrm{g} / \mathrm{g})$ y fracciones de fósforo (F1, F2, F3, F4 en $\mu \mathrm{g} / \mathrm{g}$ y \%), fósforo total $\left(\mathrm{PT}_{\mathrm{f}} \mathrm{y}\right.$ PTv en $\mu \mathrm{g} / \mathrm{g}$ ) en sedimentos del río Cuchivero, Venezuela

\begin{tabular}{|c|c|c|c|c|c|c|c|c|c|c|c|c|c|c|c|c|}
\hline $\mathrm{E}$ & $\begin{array}{l}\text { AM } \\
(\%)\end{array}$ & $\begin{array}{l}\mathrm{AF} \\
(\%)\end{array}$ & $\begin{array}{l}\text { Lodos } \\
\text { (\%) }\end{array}$ & $\begin{array}{l}\text { MOT } \\
(\%)\end{array}$ & $\begin{array}{l}\mathrm{CaCO}_{3} \\
(\%)\end{array}$ & $\begin{array}{l}\text { P-ad } \\
(\mu g / g)\end{array}$ & $\begin{array}{l}\text { P-Fe } \\
(\mu g / g)\end{array}$ & $\begin{array}{l}\text { P-F1 } \\
(\mu g / g)\end{array}$ & $\begin{array}{l}\text { P-F2 } \\
(\mu g / g)\end{array}$ & $\begin{array}{l}\text { P-F3 } \\
(\mu g / g)\end{array}$ & $\begin{array}{l}\text { P-F4 } \\
(\mu g / g\end{array}$ & $\begin{array}{l}\mathrm{PT}_{f} \\
(\mu g / g)\end{array}$ & $\begin{array}{l}\mathrm{PT}_{\mathrm{v}} \\
(\mu \mathrm{g} / \mathrm{g})\end{array}$ & $\begin{array}{l}\operatorname{Rec} \\
\%\end{array}$ & $\begin{array}{l}\text { P-I } \\
(\mu g / g)\end{array}$ & $\begin{array}{l}\mathrm{Fe} \\
(\mu g / g)\end{array}$ \\
\hline 1 & 40 & 35 & 25 & 0,87 & 2,61 & 1,87 & 3,61 & 5,49 & 0,84 & 6,28 & 50,56 & 63,16 & 63,81 & 98,99 & 12,61 & 376 \\
\hline 2 & 39 & 37 & 24 & 0,83 & 1,21 & 1,49 & 4,71 & 6,20 & 0,58 & 2,03 & 33,03 & 41,84 & 42,23 & 99,07 & 8,81 & 408 \\
\hline 3 & 45 & 40 & 15 & 0,53 & 0,89 & 1,36 & 4,71 & 6,07 & 0,71 & 2,03 & 30,86 & 39,67 & 39,90 & 99,41 & 8,81 & 427 \\
\hline 4 & 45 & 40 & 15 & 0,52 & 2,58 & 1,94 & 4,20 & 6,13 & 1,03 & 6,38 & 68,99 & 82,54 & 82,86 & 99,61 & 13,54 & 403 \\
\hline 5 & 41 & 37 & 22 & 0,76 & 0,83 & 1,87 & 4,59 & 6,46 & 0,84 & 1,84 & 27,11 & 36,24 & 36,75 & 98,61 & 9,13 & 458 \\
\hline 6 & 42 & 37 & 21 & 0,64 & 0,54 & 1,81 & 3,55 & 5,36 & 1,61 & 5,51 & 47,52 & 60,00 & 60,13 & 99,78 & 12,48 & 412 \\
\hline 7 & 38 & 39 & 23 & 0,71 & 1,35 & 1,35 & 3,49 & 4,84 & 1,10 & 4,83 & 49,28 & 60,05 & 60,19 & 99,77 & 10,77 & 428 \\
\hline 8 & 39 & 34 & 27 & 0,70 & 1,14 & 1,55 & 5,94 & 7,49 & 1,16 & 5,22 & 47,21 & 61,08 & 61,76 & 98,90 & 13,87 & 430 \\
\hline 9 & 40 & 36 & 24 & 0,69 & 1,25 & 1,36 & 5,42 & 6,78 & 0,58 & 3,58 & 28,59 & 39,52 & 40,02 & 98,76 & 10,94 & 406 \\
\hline 10 & 42 & 37 & 21 & 0,61 & 1,08 & 1,29 & 5,88 & 7,17 & 0,58 & 4,16 & 51,17 & 63,59 & 64,01 & 99,34 & 11,90 & 404 \\
\hline Min & 38 & 34 & 15 & 0,52 & 0,54 & 1,29 & 0,84 & 4,84 & 0,58 & 2,03 & 28,59 & 39,52 & 39,94 & 98,96 & 7,45 & 376 \\
\hline Max & 45 & 40 & 27 & 0,87 & 2,61 & 6,46 & 5,94 & 7,49 & 1,61 & 6,38 & 68,99 & 82,54 & 82,98 & 99,46 & 15,48 & 430 \\
\hline Prom & 41 & 37 & 22 & 0,68 & 1,41 & 2,05 & 4,24 & 6,17 & 0,91 & 4,45 & 45,25 & 56,83 & 57,06 & 99,59 & 11,53 & 410 \\
\hline DS & 3 & 2 & 4 & 0,12 & 0,71 & 0,25 & 0,97 & 0,87 & 0,35 & 1,64 & 12,68 & 14,16 & 14,17 & 0,38 & 1,85 & 17 \\
\hline
\end{tabular}




\begin{tabular}{lrrrrrrr}
\hline E & F1 & F2 & F3 & F4 & P-I & P-Fe \\
& $(\%)$ & $(\%)$ & $(\%)$ & $(\%)$ & $(\%)$ & $(\%)$ \\
\hline 1 & 8,69 & 1,33 & 9,94 & 80,04 & 19,96 & 5,72 \\
2 & 14,82 & 1,39 & 4,85 & 78,94 & 21,06 & 11,27 \\
3 & 15,30 & 1,79 & 5,12 & 77,79 & 22,21 & 11,88 \\
4 & 7,43 & 1,25 & 7,73 & 83,59 & 16,41 & 5,08 \\
5 & 17,81 & 2,32 & 5,07 & 74,80 & 25,20 & 12,65 \\
6 & 8,93 & 2,69 & 9,18 & 79,20 & 20,80 & 5,92 \\
7 & 8,06 & 1,83 & 8,05 & 82,07 & 17,93 & 5,80 \\
8 & 12,26 & 1,90 & 8,54 & 77,30 & 22,70 & 9,72 \\
9 & 17,15 & 1,47 & 9,05 & 72,33 & 27,67 & 13,72 \\
10 & 11,27 & 0,91 & 6,54 & 80,47 & 19,53 & 9,24 \\
Min & 7,43 & 0,91 & 4,85 & 72,33 & 16,41 & 5,08 \\
Max & 17,15 & 2,69 & 9,94 & 83,59 & 27,67 & 13,72 \\
Prom & 11,54 & 1,62 & 7,67 & 79,08 & 20,92 & 8,71 \\
DS & 3,55 & 0,51 & 1,80 & 3,21 & 3,21 & 3,19 \\
\hline
\end{tabular}

$\mathrm{E}=$ estación, $\mathrm{AM}=$ arena media, $\mathrm{AF}=$ arena fina, $\mathrm{CaCO}_{3}$ = carbonato de calcio, $\mathrm{P}$-ad= fósforo adsorbido, $\mathrm{P}-\mathrm{Fe}=$ fósforo absorbido a óxido de hierro, $\mathrm{F} 1=\mathrm{P}-\mathrm{ad}+\mathrm{P}-\mathrm{Fe}, \mathrm{F} 2=\mathrm{P}-$ antigénico, $\mathrm{F}_{3}=\mathrm{P}$-detrítico, $\mathrm{F} 4=\mathrm{P}$-orgánico, $\mathrm{PTf}=$ fósforo total (sumatoria de $\left.\mathrm{F} 1+\mathrm{F}_{2}+\mathrm{F}_{3}+\mathrm{F} 4\right), \mathrm{PTV}=$ fósforo total por Valderrama, \%P-I= porcentaje de fósforo inorgánico total, $\mathrm{Fe}=$ hierro, $\mathrm{P}-\mathrm{Fe}=$ fósforo enlazado a hierro, Min=mínimo, Max=máximo, Prom= promedio, Ds=desviación estándar.

\section{Resultados y discusión}

La granulometría de los sedimentos en todas las estaciones del río Cuchivero, así como la correspondiente al río Guaniamo, estuvo tipificada por un predominio de las arenas de tipo media y fina con bajos porcentajes de lodos. Las arenas medias oscilaron entre $38-45 \%$, las finas entre 34 - $40 \%$ y lodos entre 15$27 \%$. En el río Guaniamo estos valores fueron $41 \%, \quad 37 \%$ y $22 \%$ respectivamente (tabla 1).
Las concentraciones de materia orgánica total (MOT) y carbonatos fueron bajas y oscilaron entre $0,52-$ o,87\% con promedio de $0,68 \%$ y desviación estándar de 0,12 para MOT, y 0,54-2,61\%, promedio de 1,41\% y desviación estándar de 0,71 para carbonatos en el río Cuchivero. Para el río Guaniamo los valores fueron $0,76 \%$ de MOT y $0,83 \%$ de carbonatos. 
Tabla 2. Tabla de correlación de Pearson entre las concentraciones de arenas medias (AM), arenas finas (AF), Lodos, materia orgánica total (MOT); carbonato de calcio $\left(\mathrm{CaCO}_{3}\right)$, Fe y las fracciones de fósforo adsorbida más asociada a óxidos de hierro (P-F1), fracción autigénica (P-F2), fracción detrítica (P- F3), y fracción orgánica (P- F4) en sedimentos del área de estudio.

\begin{tabular}{ccccccccccc}
\hline & P-F1 & P-F2 & P-F3 & P-F4 & AM & AF & Lodos & $\mathrm{MOT}^{2} \mathrm{CaCO}_{3}$ & $\mathrm{Fe}$ \\
\hline $\mathrm{P}-\mathrm{F} 1$ & 1 & & & & & & & & & \\
$\mathrm{P}-\mathrm{F} 2$ & 0,40 & 1 & & & & & & & & \\
$\mathrm{P}-\mathrm{F} 3$ & 0,46 & 0,76 & 1 & & & & & & & \\
$\mathrm{P}-\mathrm{F} 4$ & 0,61 & 0,73 & 0,89 & 1 & & & & & & \\
$\mathrm{AM}$ & 0,58 & 0,47 & 0,48 & 0,65 & 1 & & & & & \\
$\mathrm{AF}$ & 0,54 & 0,47 & 0,66 & 0,54 & 0,99 & 1 & & & & \\
Lodos & 0,80 & 0,62 & 0,56 & 0,64 & 0,68 & 0,69 & 1 & & & \\
$\mathrm{MOT}$ & 0,75 & 0,47 & 0,53 & 0,56 & 0,74 & 0,76 & 0,94 & 1 & & \\
$\mathrm{CaCO}_{3}$ & 0,35 & 0,34 & 0,72 & 0,53 & 0,35 & 0,34 & 0,38 & 0,49 & 1 & \\
$\mathrm{Fe}$ & $-0,02$ & 0,09 & $-0,22$ & $-0,33$ & 0,01 & 0,01 & $-0,10$ & $-0,25$ & $-0,70$ & 1 \\
\hline
\end{tabular}

Se apreció correlación positiva altamente significativa entre MOT y lodos $(r=0,94)$, situación contraria mostrada por los carbonatos (tabla 2).

La cuenca del río cuchivero situada en la parte occidental del Escudo Guayanés, discurre por la provincia estructural que recibe su mismo nombre, la cual expone extensamente rocas plutónicas, epimeso y meso-zonales, con vestigios mucho menos voluminosos de rocas meta-volcánicas de composición predominantemente silíceas y metasedimentarias de disposición clásticas (17). Los bajos niveles de MOT son debidos a la baja capacidad de las arenas para adsorber la materia orgánica. Por otra parte, no evidencian impactos antropogénicos ya que son muy inferiores al valor de $20 \%$ indicado para zonas impactadas (18).

Sedimentos de los ríos Cuchivero y Guaniamo mostraron distribuciones bastantes homogéneas con variaciones estándar inferiores a cuatro, sin embargo, la preponderancia de arenas y bajos contenidos de MOT y carbonatos, evidencian un lavado constante de los edimentos debido a la alta energía de las aguas de este sistema fluvial.

La concentración de la sumatoria de la fracción de fósforo intercambiable más asociado a óxidos de hierro (F1) mostró buena correlación con el menor tamaño de la partícula ( $\mathrm{r} \geq 0,80$ ), tabla 2. En el río Cuchivero la concentración osciló entre 4,84 $\mu \mathrm{g} \mathrm{P/g} \mathrm{(1,35} \mathrm{de} \mu \mathrm{g}$ Pad $/ \mathrm{g}+3,49 \mu \mathrm{g}$ P-Fe/g) en la estación siete y 7,49 $\mu \mathrm{g} \mathrm{P} / \mathrm{g}(1,55 \mu \mathrm{g}$ P- ad /g $+5,94 \mu \mathrm{g}$ P-Fe/g) en la estación 8.

En el resto de las estaciones los valores presentaron poca variación y se ubicaron entre estos rangos de concentraciones.

El promedio fue de $6,17 \mu \mathrm{g} P / g$ y la desviación estándar de 0,87 . En el río Guaniamo el valor fue de 6,46 $\mu \mathrm{g} \mathrm{P} / \mathrm{g}$ 


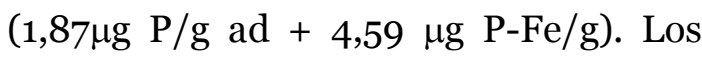
resultados indican que indica que el mayor componente de esta fracción, lo representa el P-Fe. La contribución de la fracción $\mathrm{F} 1$, al fósforo fue de 11,54\% y 17, $81 \%$ para el Cuchivero y Guaniamo respectivamente (tabla 1, figura $2 \mathrm{~A}$ ). La fracción autigénica (F2) presentó un promedio de $0,91 \mu \mathrm{g} \mathrm{P} / \mathrm{g}$ y desviación estándar de 0,35 (tabla 1). La concentración de esta fracción mostró mejor correlación con los lodos ( $\mathrm{r}=0,62$, tabla 2$)$.

Figura 2. Distribución espacial de las diferentes fracciones de fósforo (2A). Comportamientos inversos de las concentraciones de hierro - fósforo enlazado a hierro (2B), fósforo orgánico - fósforo enlazado a hierro (2C), y hierro - fósforo orgánico (D) en el ecosistema bajo estudio

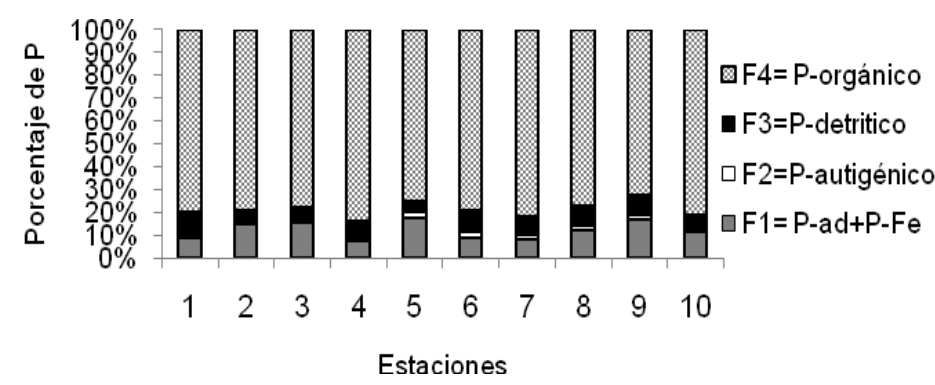

A
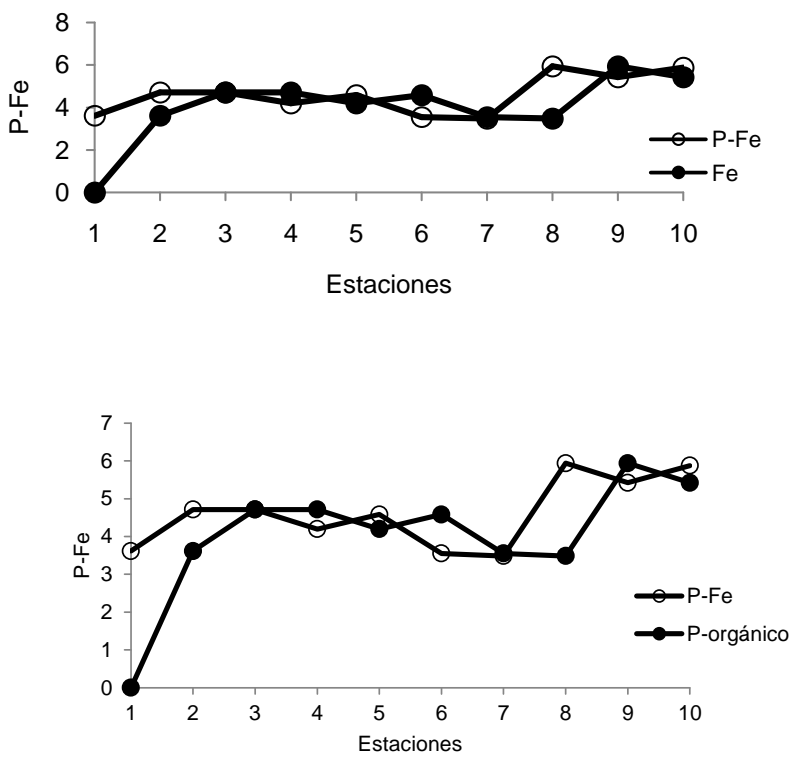

$\mathrm{C}$

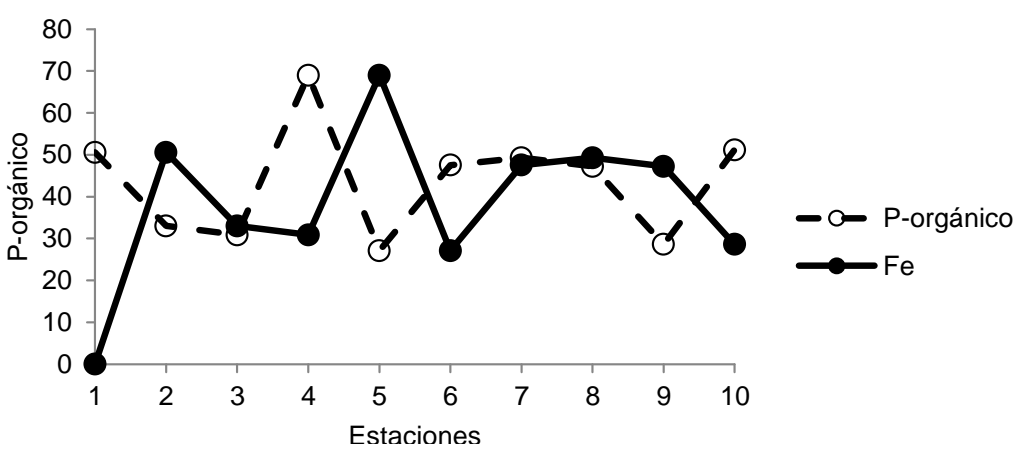

$\mathrm{D}$ 
Los valores variaron desde $0,58 \mu \mathrm{g}$ $\mathrm{P} / \mathrm{g}$ en las estaciones dos, nueve y diez, hasta 1,61 $\mu \mathrm{g} \mathrm{P/g}$ en la estación seis. En el río Guaniamo el valor fue de $0,84 \mu \mathrm{g} \mathrm{P} / \mathrm{g}$. La contribución al fósforo total de esta fracción de $\mathrm{P}$ total fue de 1,62\% y 2,32\% para el Cuchivero y Guaniamo respectivamente (tabla 1 , figura $2 \mathrm{~A}$ ).

La distribución del fósforo detrítico osciló entre 2, $03 \mu \mathrm{g} \mathrm{P} / \mathrm{g}$ en las estaciones dos y tres hasta $6,38 \mu \mathrm{g} \mathrm{P} / \mathrm{g}$ la cuatro. El promedio fue de 4,45 $\mu \mathrm{g} \mathrm{P} / \mathrm{g}$ y la desviación estándar de 1,45. En el río Guaniamo el valor fue de $1,84 \mu \mathrm{g} \mathrm{P} / \mathrm{g}$ (tabla 1).

La concentración de esta fracción mostró de mejor correlación con las arenas finas $(\mathrm{r}=0,66$, tabla 2). La contribución al fósforo total de esta fracción de P total fue de 7,67\% y 5,07\% para el Cuchivero y Guaniamo respectivamente (tabla 1 , figura $2 \mathrm{~A}$ ).

La fracción cuatro $\mathrm{u}$ orgánica(F4) presentó un valor promedio para el río Cuchivero de 45,25 $\mu \mathrm{g} \mathrm{P/g} \mathrm{(28,59} \mathrm{\mu g} \mathrm{P/g} \mathrm{-68,99} \mathrm{\mu g}$ $\mathrm{P} / \mathrm{g}$ ), y desviación estándar de 12,68, mientras que en el río Guaniamo el valor encontrado fue $27,11 \mu \mathrm{g} \mathrm{P} / \mathrm{g}$.

La máxima concentración fósforo orgánico se determinó en la estación cuatro, al igual que ocurre con el detrítico, lo cual puede estar influenciada por la deposición causada por el rebalse que se forma en esa zona debido a la unión del río Guaniamo con el Cuchivero. La fracción orgánica mostró correlación significativa $\mathrm{r}=0,64$ con los lodos. $\mathrm{La}$ contribución al fósforo total de esta fracción de $\mathrm{P}$ total fue de 79,08\% y 74, 80\% para el Cuchivero y Guaniamo respectivamente (tabla 1, figura 2A).
El promedio de fósforo total $\left(\mathrm{PT}_{\mathrm{f}}\right)$ para el río Cuchivero fue de $56,83 \mu \mathrm{g} \mathrm{P} / \mathrm{g}$ con una desviación estándar de 14,16. El valor mínimo $(39,52 \mu \mathrm{g} \mathrm{P} / \mathrm{g})$ se detectó en la estación nueve y el máximo $(82,54 \mu \mathrm{g}$ $\mathrm{P} / \mathrm{g}$ ) en la estación cuatro respectivamente. En el río Guaniamo el valor fue $36,24 \mu \mathrm{g} \mathrm{P} / \mathrm{g}$ (tabla 1). El $\mathrm{PT}_{\mathrm{f}}$ mostró un patrón de distribución concordante con lo apreciado para la fracción orgánica, la cual fue la de mayor valor.

Las características observadas en las distribuciones de todas las fracciones de fósforo en la zona de estudio sugieren la influencia de diferentes mecanismos de transporte y mecanismos posdepositacionales de partículas de composición química variable. Los altos porcentajes de arenas en comparación con los lodos evidencian un lavado constante de los sedimentos en el ecosistema estudiado, lo cual es un reflejo de la alta energía en este este sistema fluvial.

El fósforo orgánico, a pesar de que el contenido de materia orgánica fue bajo, representó en promedio el $79,08 \%$ y 74,80\% del fósforo total los ríos Cuchivero y Guaniamo respectivamente (tabla 1 , figura $2 \mathrm{~A}$ ). La gran área superficial que posee la materia orgánica, permite la fijación del fósforo en su estructura, permitiendo su acumulación. Las arenas que fueron mayoritarias básicamente constituidas por partículas de cuarzo, no poseen cargas negativas superficiales como los limos, arcillas y la MOT, que pudieran facilitar la interacción y adsorción del 
$\mathrm{P}$ en sus partículas mientras se movilizan en la columna de agua (19). Las excreciones de la fauna béntica, peces y del zooplancton, junto con la degradación bacteriana de materia orgánica continental, son factores que pueden jugar un papel importante en la distribución de la fracción orgánica en el sedimento de la zona bajo estudio. Los valores de $\mathrm{P}$ orgánico son importantes ya que reflejan el estado trófico del ecosistema y pudiera sugerir que en la zona, la producción orgánica (producción primaria) es moderadamente alta y que parte de ese fósforo podría estar siendo traslocado hacia oxihidróxidos de hierro tras la transformación de la materia orgánica (20). Tal situación ha sido evidenciada en el río Misisipi (2). Los valores de P- orgánico determinados son superiores a las concentraciones de $10,94 \mu \mathrm{g} \mathrm{P} / \mathrm{g}$, 4,29 $\mu \mathrm{g} \quad \mathrm{P} / \mathrm{g} \quad \mathrm{y} \quad 2,47 \quad \mu \mathrm{g} \quad \mathrm{P} / \mathrm{g}$ determinados para el rio Misisipi, río Amazonas y para la Costa Atlántica Venezolana respectivamente $(2,21$, 1).Estos ríos son de mayor caudales, y están más expuestos a las actividades humanas, lo cual pudiera sugerir que los valores en el Cuchivero y Guaniamo son autóctonos de estos ecosistema.

Parte de fracción orgánica tiene origen en material orgánico de animal o vegetal autóctono de la cuenca del Cuchivero, Los bajos valores de materia orgánica, no vislumbra incorporación antropogénica de materia orgánica, puesto que los valores son inferiores a 20\%. Zonas afectadas por materia orgánica, son aquellas que presentan contenidos iguales o superiores al $20 \%$
(18). La fracción P-ad + P- Fe, fue la segunda en magnitud con porcentaje promedio de $11,54 \%$ para el río Cuchivero los cuales corresponden a $2,83 \%$ de $\mathrm{P}$-ad $+8,71 \% \quad \mathrm{P}-\mathrm{Fe}$ respectivamente, poniendo de manifiesto que una parte del fósforo total puede ser desorbido o intercambiado dentro del sedimento e incorporarse a la columna de agua. Esto sugiere mecanismos intensos en la transformación hacia las fracciones $\mathrm{P}$-ad + P- Fe (1). Concentraciones altas fósforo asociado a hierro (P-Fe) proveen información de aéreas impactadas antropogénicamente, mientras que el asociado a apatita biogénica representa un indicador de la influencia de cultivos de peces sobre los sedimentos fluviales $\mathrm{y}$ costeros $(22,23)$.

El P-Fe mostró un patrón de distribución inverso con el Fe y con el P-orgánico (Figura 2B, 2C). Esto pudiera ser debido a que en primer lugar, el Fe sería removido para la formación del P-Fe, y en segundo lugar porque el hierro posteriormente seguiría agotándose al ser utilizado como aceptor de electrones para degradar la materia orgánica. Durante esta degradación es liberando fosfato y metabolitos orgánicos, estos últimos podrían posteriormente mostrar mecanismos de sorción o reasorción sobre las partículas detríticas de la materia orgánica recalcitrante, tal como se muestra en la figura 2D (24).

La cantidad total de $\mathrm{P}$ biodisponible en el ecosistema bajo estudio, representado por la sumatoria de F1+ F4 que son las fracciones más propensas a degradación. 
La suma de estas fracciones equivalente a un valor promedio de $51,41 \mu \mathrm{g} \mathrm{P} / \mathrm{g}$, representando el 90,47\% del PT (tabla 1). La posible descomposición microbiana de la materia orgánica sedimentaria y $\mathrm{P}$ biodisponible en los ríos Cuchivero y Guaniamo, pudieran resultar en la liberación de fosfato para la columna de agua donde pudiera estar disponible para la producción primaria, sin embargo, podría pero plantear un amenaza de eutrofización en las aguas a futuro (25).

El porcentaje de P-autigénico fue 1,62 \% y bajo en comparación con los valores de 3,61, 4,39 de los ríos Amazonas y Misisipi y respectivamente (21). La distribución de esta fracción refleja la presencia de carbonato fluorapatita de origen autigénico (CFA), apatita biogénica la cual incluye $\mathrm{P}$ de restos de los peces) y $\mathrm{P}-\mathrm{CaCO}_{3}$ o fosforo asociado a carbonato (1). Este último confirmado por los bajos valores de carbonato de calcio (tabla 1). El P-autigénico refleja el grado $o$ intensidad de las transformaciones del fósforo en el sedimento por procesos diagenéticos durante su enterramiento, especialmente en la diagénesis temprana (1). Numerosos estudios han indicado que una significante reorganización diagenética del fósforo ocurre durante la etapa de enterramiento, especialmente en sedimentos de márgenes continentales (26). La salinidad afecta la química del fósforo refractario que es enterrado en el sedimento; es por ello que en aguas fluviales hierro y fósforo pueden ser enterrados como vivianita $\left(\mathrm{FePO}_{4} 28 \mathrm{H}_{2} \mathrm{O}\right)$, estregnita $\left(\mathrm{FePO}_{4} \cdot 2 \mathrm{H}_{2} \mathrm{O}\right) \mathrm{u}$ otros compuestos férricos, los cuales no podrían formarse en aguas salobres donde el Fe (II) se combina con los sulfuros (27).

El porcentaje promedio de $\mathrm{P}$ detrítico fue de $7,67 \%$ para el río Cuchivero. La fracción detrítica que es de origen litogénico continental o terrestre es también llamado fósforo asociado a apatita, y en los ecosistemas fluviales es afectado por las cargas de sedimentos terrígeno de la cuenca, carbonato biogénico, lodos (1). El P- detrítico en el río Cuchivero presentó correlación significativa con el P-orgánico $(\mathrm{r}=0,89)$ y con $\mathrm{P}$ autigénico y carbonatos $(\mathrm{r}=0,76$ y $\mathrm{r}=0,72$ ), tabla 2, lo cual representa asociación entre estas formas del fósforo, esto sugiere que estás fracciones son afectadas por los mismos procesos químicos o mecanismos de reacomodo posdeposicionales. Esta fracción geológicamente ha sido creada por procesos ígneos y metamórficos (28). La cuenca del Cuchivero se encuentra situada en la parte occidental del Escudo Guayanés, y discurre sobre rocas plutónicas, epi-meso y mesozonales, con vestigios de rocas metavolcánicas de composición predominantemente silíceas y metasedimentarias de disposición clásticas (17). El promedio de $\mathrm{P}$ detrítico determinado en esta investigación $(4,45 \mu \mathrm{g} \quad \mathrm{P} / \mathrm{g})$ es comparable al valor $(5,16 \mu \mathrm{g} / \mathrm{P})$ reportados por para el río Misisipi (2) y para la concentración de 4,65 $\mu \mathrm{g} \mathrm{P/g}$ de la Costa Atlántica Venezolana, la cual está influenciada por la descarga del río Orinoco (1). 


\section{Conclusiones}

Las diferentes fracciones del fosforo se asociaron con el menor tamaño de la partícula del sedimento. La mayor contribución al fósforo total en la zona de estudio proviene del fósforo orgánico alóctono, cuyo origen puede ser, las excreciones de la fauna béntica, peces y del zooplancton, junto con la degradación bacteriana de materia orgánica continental, en vista de los bajos valores de materia orgánica total.

Esta fracción en conjunto con el $\mathrm{P}$ intercambiable o adsorbido, $\mathrm{y}$ el fósforo enlazado a hierro, representan más del $90 \%$ del $\mathrm{P}$ que se encuentra totalmente biodisponible para la biota. Por otra parte $8,71 \%$ de P-Fe el cual ha sugerido en parte de origen antropogénico, no refleja riesgo para el ecosistema.

La presencia de P-autigénico sugiere mecanismos y reacciones en la cual el fósforo posiblemente es transformado en forma orgánica. Por otra parte, los bajos valores de la fracción detrítica refleja baja meteorización de las rocas que cubren la cuenca del río Cuchivero.

El porcentaje promedio de $\mathrm{P}$ biodisponible fue $51,41 \mu \mathrm{g} \mathrm{P} / \mathrm{g} \mathrm{P}$, lo cual representa el 90,47\% del $\mathrm{PT}_{\mathrm{f}}$. El contenido de esta forma en el sedimento podría ser una potencial fuente de $\mathrm{P}$ hacia la columna de agua que conseguiría ser liberado bajo determinadas condiciones de óxidoreducción, pudiendo tener a futuro un impacto sobre el ecosistema de los ríos Cuchivero y Guaniamo.
Referencias bibliográficas

1. MÁRQUEZ A., GUILLOT L., SENIOR W., MÁRTINEZ G., GONZÁLEZ Á. Bol Inst Oceanogr Vzla 53 (1): 111122. 2014.

2. 2. SUTULA M., BIANCHI T. S., MCKEE B. A. Limnol Oceanogr 49 (6): 2223-2235.2004.

3. BENÍTEZ -NELSON C. R. Ciencia al Día 3: 1-14.1990.

4 BLAKE L., HESKETH N., FORTUNE S., BROOKES P. Soil Use Manage; 18:199-207. 2002.

5. MOKAYA S., MATHOOKO J., LEICHTFRIED M. Afr $J$ Ecol. 42: 281288.2004.

6. SONG G., LIU S. Acta Oceanol Sin 34: 4, 84-91.2015.

7. SPIVAKOV B., MARYUTINA T. A., MUNTAU H. Pure Appl Chem 71:11, 2161-2176. 1999.

8. EIJSINK L., KROM M., HERUT B. Amer J Sci 300: 483-503.2000.

9. ANDERSON L., DELANEY M. Limnol Oceanogr 45 (2): 509-515. 2000.

10.http://www.buenastareas.com/ensayo s/Rio-Cuchivero/1749357.html. Fecha de consulta. 13/6/2015

11. VALDERRAMA J. M Chem 10: 109122.1981

12. MURPHY J., RILEY H. An Chim Acta 12: 162-170. 1962.

13.MÁRQUEZ A., SENIOR W., MARTÍNEZ G., GONZÁLEZ Á. Ciencia 17 (1): 87 97. 2009.

14.GONZÁLEZ H., RAMIREZ M. J Geochem Explore 52 (1-2): 183192.1995 .

15.VOGEL A. 1960. Química Analítica Cuantitativa. Vol. 1. (Eds). Kapelusz, S.A Buenos Aires (Argentina). 811 pp. 1995.

16. LEWIS B., LANDIG W. Mar Chem 40: 105-14. 1992.

17.COLVEÉ P, SZEZERBAN E., TALUKDAR S. Estudio $y$ consideraciones geológicas sobre la cuenca del río Caura. 
En: WEIBEZAHN F., ALVAREZ H., LEWIS JR W (Eds). El rio Orinoco como ecosistema. Caracas, Venezuela. pp. 430.1990

18.PÁEZ-OSUNA F., BOTELLO A., VILLANUEVA S. Mar Poll Bull 34(2): 123-128.1986

19. ROJAS N., SILVA N. Cien Tecnolog Mar 26(1): 15-31. 2003.

20.JORDAN T., CORNWELL J., BOYNTON W., ANDERSON T. Limnol Oceanogr 53(1), 172184.2003.

21.BERNER R., RAO J. Geochim

Cosmochim Acta 58: 2333- 2339. 1994.

22.MATIJEVIÆ S., KLJAKOVIC-GAŠPIE Z., BOGNER D., GUGIE A., MARTINOVIC I. Adriat Acta 49: $165-184.2008$.

23.RUTTENBERG K.C. Geochim Cosmochim Acta 57, 9911007.1993.

24.CHRISTOPHORIDIS C., FYTIANOS K. J Environ Quality 35(4), 11811192.2006.

25.ROZAN T.F., TAILLEFERT M., TROUWBORST R.E. Limnol Oceanogr 47(5):1346-1354.2002.

26. FILIPPELLI G. Mar Geol. 174: 307321.2001

27. GÄCHTER R., MÜLLER A. Limnol Oceanogr Notes 48: 929-933. 2003.

28.COLLIS H. 2006 Application of shortterm sediment dynamics and particlebound phosphorus fractionation methods (SEDEX) to estimate the benthic nutrient loading potential in upper Newport estuary, California. (Para obtener el título de Maestría), Pacific University, USA, 96pp. 2006. 


\section{UNIVERSIDAD}

DEL ZULIA

\section{$\mathbb{C}\|\mathbb{N} \mathbb{N}\| \mathbb{A}$}

Vol. 24, No 2(2016)

Esta revista fue producida y editada en formato digital en marzo de 2016, por el personal de la Revista $\mathbb{C} \| \mathbb{E} \mathbb{N} C \mathbb{A}$, Oficina de Publicaciones Científicas de la Facultad Experimental de Ciencias, Universidad del Zulia.

Maracaibo - Venezuela

www.luz.edu.ve

www.serbi.luz.edu.ve

produccioncientifica.luz.edu.ve 


\section{$(\mathrm{C}][\mathrm{E} \mathbb{N}(\mathrm{C}][\mathrm{Al}$ \\ Revista Científica de la Facultad Experimental de Ciencias de la Universidad del Zulia

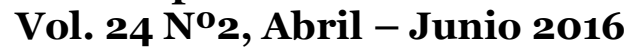

Pg.

\section{BIOLOGÍA/BIOLOGY}

Mejoramiento microbiológico de ratones convencionales BIOU:NMRI usando la técnica de Histerectomía

Microbiological improvement of BIOU:NMRI conventional mice by Technology of Hysterectomy

Adán Galúe y Rosa De Jesús

(Mérida, Venezuela)

Constituyentes químicos y actividad antiinflamatoria de Marcetia taxifoliade Marcetia taxifolia

Chemical constituents and anti-inflammatory activity of Marcetiataxifolia

Jani Baptista, Katiuska Chávez, Fátima Torrico, Ernesto Trejo, Carmen C. Garcia, Jensaida

Urbina, José Carrasco, Antonieta Taddei, Stephen Tillett y Alírica I. Suárez (Caracas , Venezuela)

\section{FÍSICA/ PHYSICS}

Análisis de observaciones espectroscópicas de dos estrellas post-AGB: Determinación de la velocidad radial

Analysis of spectroscopic observations of two post-AGB stars: Determination of their radial velocity

Patricia Rosenzweig L, Gabriela García Lugo, José A. Meléndez F., Wilmer Useche, María-

Teresa Celis y Dan Pooley

(Mérida, Venezuela)

\section{QUÍMICA/ CHEMISTRY}

Validation of a GC-MS method for the simultaneous determination of five coumarins derivatives in natural soil samples

Validación de un método por CG-EM para la determinación simultanea de cinco derivados de cumarina en muestras de suelo natural

Alberto de J. Oliveros-Bastidas, Luiz Claudio de Almeida Barbosa and Antonio J. Demuner (Caracas, Venezuela)

Especiación del fósforo en sedimentos de un río tropical venezolano y su posible impacto sobre el ecosistema Phosphurus speciation in sediments of a Venezuelan tropical river and its posible impacto $n$ the ecoystem 